\title{
La chétotaxie des larves nageantes de Polystomes européens (Monogenea)
}

\author{
par Cl. COMBES et A. LAMBERT \\ (collaboration technique: M.-Th. ALmÉRAs) \\ Département de Biologie animale, Centre Universitaire, $F 66025$ Perpignan, \\ et Laboratoire de Parasitologie comparée, U.S.T.L., F 34060 Montpellier Cedex
}

\section{Résumé.}

Les sensilles des larves nageantes des Monogènes du genre Polystoma peuvent être classées en groupes et en cycles transversaux.

L'étude de trois espèces européennes montre que les deux générations de larves issues, l'une de l'adulte, l'autre du néoténique, ont la même chétotaxie, que la variation individuelle est extrêmement limitée et que certaines espèces voisines peuvent être distinguées par des variations de détail sur un schéma fondamental.

\section{Summary.}

Chaetotaxy of the swimming larvae of European Polystomes.

The sensillae of the swimming larvae of the Monogeneans of the Polystoma genus can be classified into groups and transversal cycles.

The study of three european species shows that the two generations of larvae, one of them coming from the adult, the other one from the neotenic, have the same chetotaxy, that there are very few individual differences from one larva to the next, and that closely related species can sometimes be distinguished by minor variations within a fundamental pattern.

Nous entendons par chétotaxie la disposition des soies sensorielles (ou sensilles) sur le tégument des Plathelminthes. Cette chétotaxie a fait jusqu'ici l'objet d'études plus détaillées chez les Digènes que chez les Monogènes. Ainsi J. Richard (1971) a proposé un système cohérent pour l'étude chétotaxique des cercaires. Il s'est avéré que la disposition des sensilles est un caractère à faible variabilité intra-spécifique, 
donc précieux pour la détermination des stades larvaires; il permet, en outre, d'affirmer ou de confirmer les parentés phylétiques des différentes espèces ou des différents groupes taxonomiques. Dans le cas des cercaires de Digènes, il est désormais possible de préciser de nombreuses attributions familiales par le seul examen de la chétotaxie.

En ce qui concerne les Monogènes, les larves nageantes de quelques espèces ont vu leur chétotaxie décrite avec plus ou moins de précision: c'est le cas de Tetraonchus monenteron (Wagener, 1857) d'après B. E. Bychowsky (1957), de trois Polystomatidae d'Europe d'après C. Combes (1968), de trois Polystomatidae d'Afrique d'après A.-M. Maeder (1973), de Discocotyle sagittata (Leuckart, 1842) Diesing, 1850, d'après I. L. Owen (1970) et de Diplozoon paradoxum Von Nordmann, 1832 d'après Euzet et Lambert $(1971,1974)$.

Cependant, aucun système cohérent pour l'étude chétotaxique des larves de Monogènes n'a été utilisé jusqu'ici.

Dans le présent travail, nous donnons une description précise de la disposition des soies sur les larves de Polystomatidae. Nous fondons notre étude sur trois espèces européennes :

- Polystoma integerrimum (Fröhlich, 1798) parasite de Rana temporaria L. ; (Cuv.) ;

- Polystoma pelobatis (Euzet et Combes, 1966) parasite de Pelobates cultripes

- Polystoma gallieni Price, 1938 parasite de Hyla meridionalis Boettg.

Pour la description de la chétotaxie, nous proposons une classification et une numérotation dans le but d'aider à dégager les caractères ayant une valeur générale et de mettre en évidence les variations spécifiques.

\section{Classification des sensilles :}

Pour permettre d'éventuelles comparaisons, nous avons cherché à nous inspirer du système proposé par J. Richard (1971) pour les cercaires de Digènes.

Richard divise la cercaire-type en zones: céphalique, préacétabulaire, acétabulaire, post-acétabulaire, caudale et éventuellement furcale.

Dans chaque zone, elle considère qu'il existe des commissures nerveuses transversales reliant entre eux six grands troncs longitudinaux (2 ventraux, 2 latéraux et 2 dorsaux). En théorie, les sensilles seraient placées aux intersections des commissures transverses et des troncs longitudinaux.

Chez les larves nageantes de Monogènes, ce que l'on sait du système nerveux autorise à fonder une classification des sensilles sur un schéma d'inspiration comparable : en effet, le travail de K. Rohde (1968), décrit le système nerveux d'un Polystomoïdes adulte comme composé également de six troncs nerveux longitudinaux réunis par de nombreuses commissures transverses. Il est donc possible, dans le cas 
des Monogènes, comme dans celui des Digènes, de parler de sensilles dorsales, correspondant aux deux tronzs longitudinaux dorsaux, de sensilles latérales correspondant aux deux troncs longitudinaux latéraux et de sensilles ventrales correspondant aux deux troncs longitudinaux ventraux. Il est également possible de distinguer, d'avant en arrière, une série de cycles transversaux de sensilles dont on peut supposer qu'ils correspondent aussi aux intersections de commissures transverses.

Par contre, il va de soi qu'on ne saurait conserver les zones proposées par J. Richard, la morphologie d'une larve nageante de Monogène étant fondamentalement différente de la morphologie d'une cercaire de Digène.

Nous rappellerons brièvement qu'une larve nageante de Polystomatidae comprend un corps prolongé par un hapteur postérieur et porteur de groupes de cellules ciliées; celles-ci au nombre de 55 sont disposées de la manière suivante (fig. 1 et 2):

Groupe apical (fig. $1, A$ ): une seule cellule, médiane et impaire.

Groupe céphalique (fig. $1, B$ ) : $2 \times 12$ cellules, dorsales et ventrales.

Groupe médio-antérieur (fig. 1, C) : $2 \times 3$ cellules, toutes ventrales.

Groupe médio-postérieur (fig. 1, D) : $2 \times 6$ cellules, dorsales et ventrales.

Groupe haptorial (fig. 1, E): $2 \times 6$ cellules, toutes dorsales.

Nous proposons de diviser la larve de Monogène en trois zones principales (fig. 1 et 2):

- Une zone antérieure ou céphalique comprenant la région péribuccale jusqu’à la limite antérieure du groupe cilié céphalique; la région ainsi définie englobe la cellule apicale,

- Une zone moyenne ou troncale comprenant la région située entre le bord antérieur du groupe cilié céphalique et la concavité ventrale du hapteur; cette région englobe donc la totalité des cellules ciliées, exception faite de la cellule apicale.

- Une région postérieure ou haptoriale représentée par la face concave du hapteur.

A l'intérieur de ces zones, nous distinguerons, suivant le cas, des groupes et des cercles de sensilles (fig. 1 et 2 ).

La zone céphalique comprend: 1 groupe jugal, symbolisé par $\mathrm{Ju} ; 1$ groupe apical, symbolisé par Ap; 2 cycles de sensilles buccales numérotés Bu1 et Bu2; 2 cycles de sensilles labiales numérotés La1 et La2.

La zone troncale comprend: 9 cycles numérotés de $\operatorname{Tr} 1$ à $\operatorname{Tr} 9$.

$\operatorname{Tr} 1$ à $\operatorname{Tr} 5$ sont situés entre le groupe cilié céphalique et le groupe cilié médioantér:eur.

Tr6 entre le groupe cilié médio-antérieur et le groupe cilié médio-postérieur.

$\operatorname{Tr} 7, \operatorname{Tr} 8$ et $\operatorname{Tr} 9$ entre le groupe cilié médio-postérieur et le groupe cilié haptorial.

La zone haptoriale comprend: 3 cycles numérotés $\mathrm{Ha} 1, \mathrm{Ha} 2$ et $\mathrm{Ha} 3$.

Le cycle Ha1 est le cycle le plus périphérique.

Le cycle $\mathrm{Ha} 3$ est le plus central. 


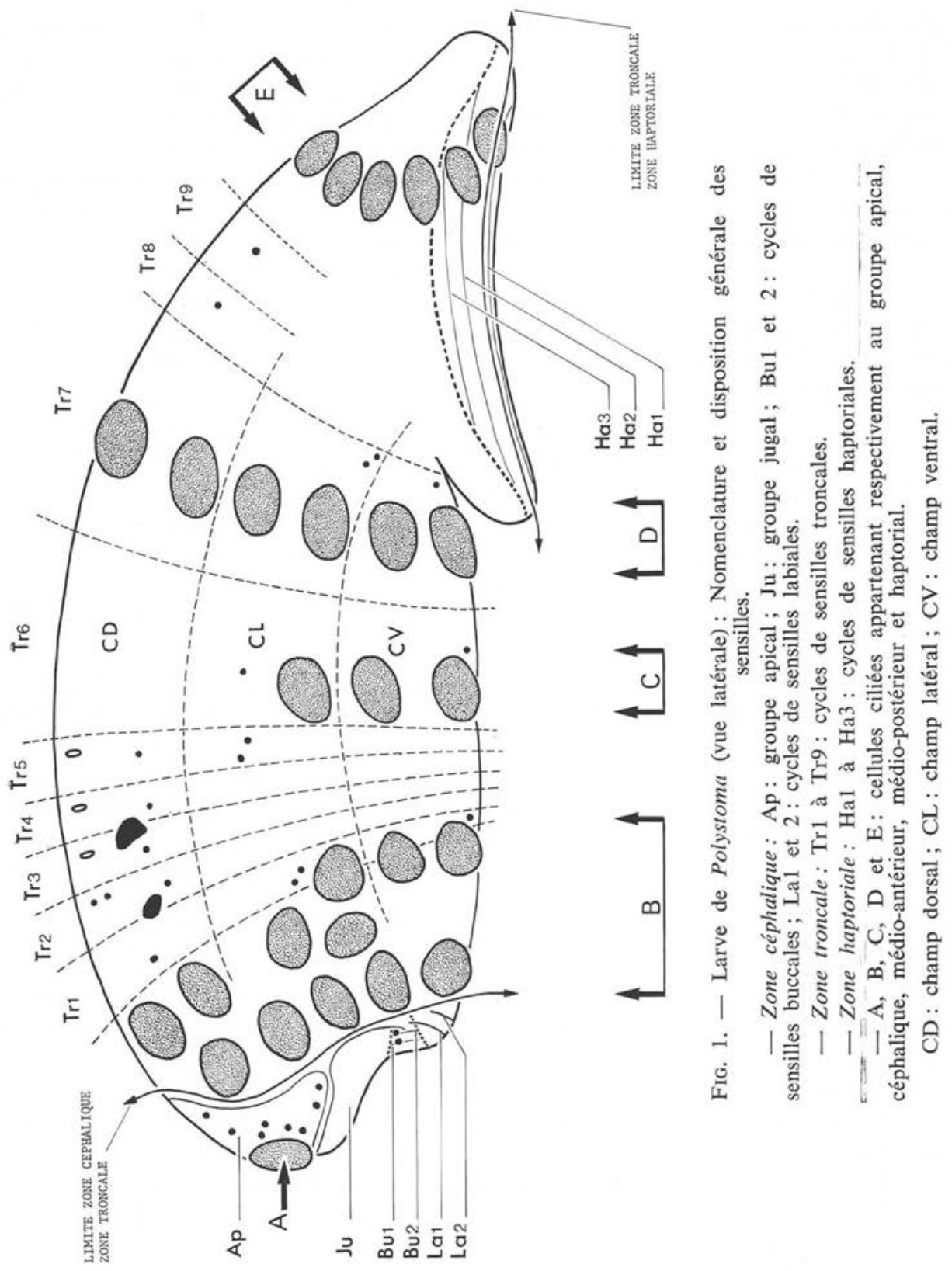




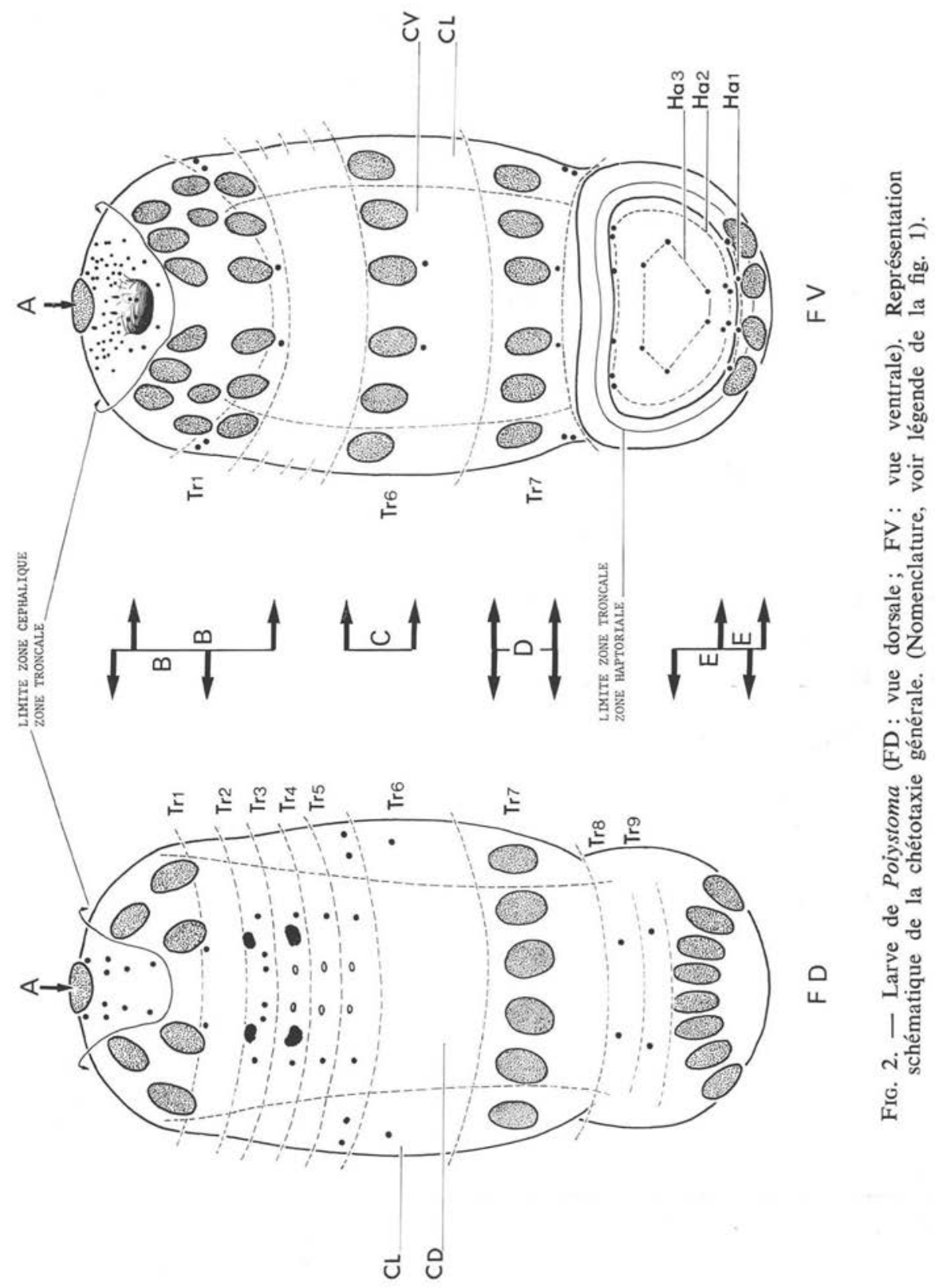


Dans chacun des cycles, les sensilles sont désignées par une lettre $\mathrm{D}, \mathrm{L}$ ou V exprimant leur situation vis-à-vis des axes nerveux longitudinaux, suivant qu'elles dépendent respectivement des axes dorsaux, latéraux ou ventraux (fig. 1 et 2).

La description est toujours faite pour un hémicorps, la disposition des sensilles étant symétrique à un plan sagittal. La figure 1 montre sur une larve schématisée, de profil, la distinction en trois zones et la disposition des cycles transversaux vis-à-vis des groupes ciliés.

Pour chaque portion d'un cycle (dorsale, latérale ou ventrale) nous préciserons le nombre de sensilles correspondantes:

Par exemple: «Cycle $\operatorname{Tr} 7: 0 \operatorname{Tr} 7 \mathrm{D}, 2 \operatorname{Tr} 7 \mathrm{~L}, 1 \mathrm{Tr} 7 \mathrm{~V}$ », signifie qu'il n'y a pas de sensille dorsale $(0 \operatorname{Tr} 7 \mathrm{D})$, qu'il y a 2 sensilles latérales $(2 \operatorname{Tr} 7 \mathrm{~L})$ et 1 sensille ventrale $(1 \operatorname{Tr} 7 \mathrm{~V})$ pour le cycle troncal numéro 7.

\section{Chétotaxie :}

La description que nous donnons ci-après doit être considérée comme une synthèse des observations effectuées sur les trois espèces étudiées (les particularités de chaque espèce sont analysées au paragraphe suivant).

\section{1) Zone céphalique.}

a) Groupe jugal (fig. 3, 4, 5 et 6).

Le groupe jugal est le plus délicat à interpréter en raison de ses caractéristiques mêmes:

- les sensilles y sont nombreuses (26 à 38) et serrées ;

- leur situation les unes par rapport aux autres est variable ;

- certaines d'entre elles possèdent plusieurs soies au lieu d'une ;

- il n'est pas exclu que des structures autres que les sensilles soient ici imprégnées par l'argent (orifices de glandes notamment).

On retiendra que :

- l'ensemble du groupe est situé dorso-latéralement par rapport à la lèvre de l'entonnoir buccal; sa limite dorsale est toujours voisine du niveau du bord ventral de la cellule apicale;

Fig. 3, 4, 5 et 6. - Chétotaxie de la zone céphalique des Polystomes européens, dessinés à la chambre claire, à la même échelle. (Nomenclature: voir légende de la fig. 1). $\mathrm{a} 1, \mathrm{a} 2, \mathrm{~b} 1, \mathrm{~b} 2$ et $\mathrm{b} 3$ : sensilles particulières du groupe jugal (voir texte).

3. - Polystoma integerrimum. 4. - P. pelobatis. 5. $-P$. gallieni, 6, $-P$. integerrimum (région frontale aplatie). 


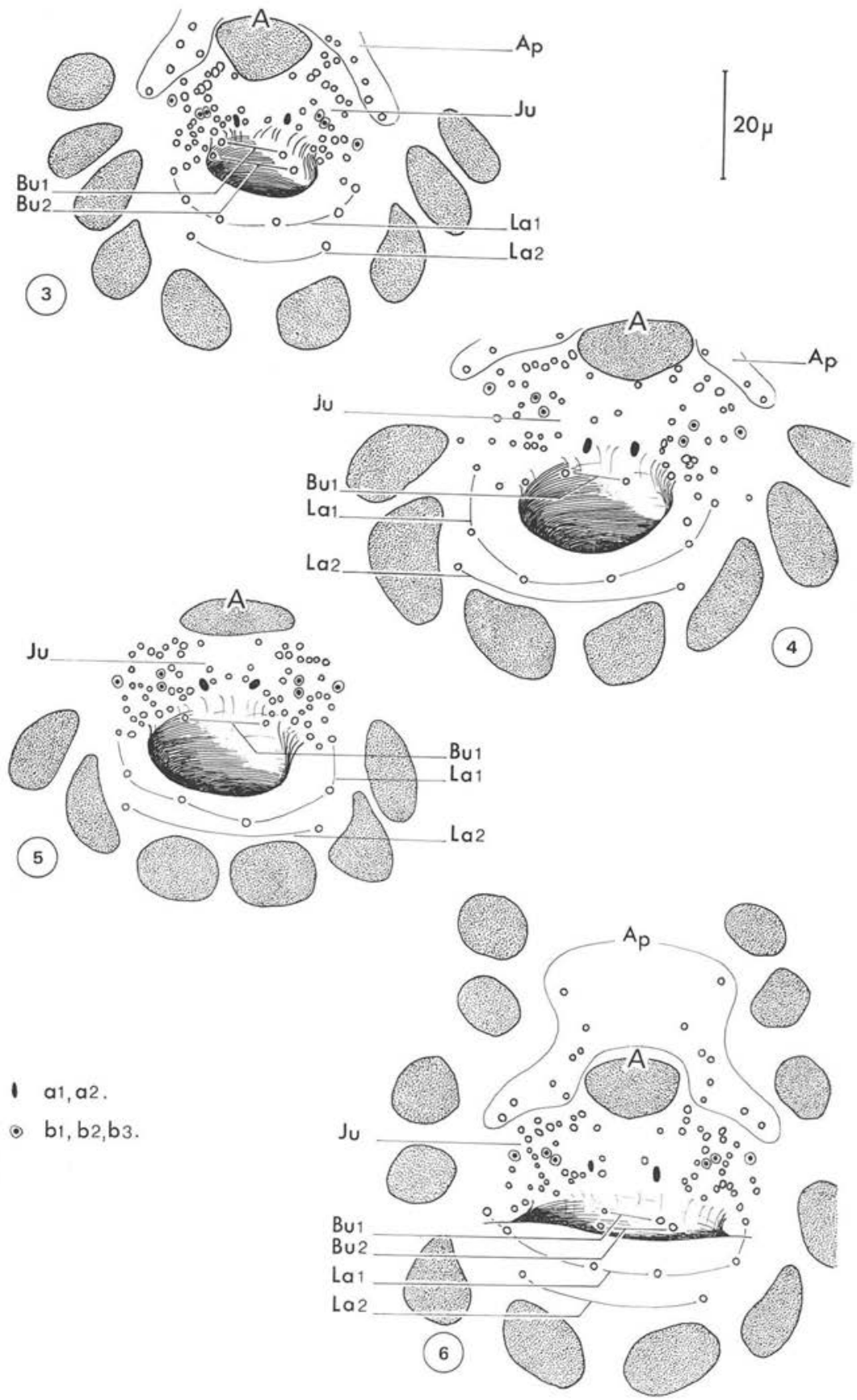


- près de la ligne médiane et sur le rebord même de la bouche se trouvent des sensilles de grande taille (fig. 3, 4, 5, 6: a1 et a2) qui portent chacune plusieurs soies courtes ;

- vers le centre du groupe on observe régulièrement trois structures argyrophiles, de taille supérieure aux sensilles normales, qui représentent soit, comme les précédentes, des sensilles d'un type particulier, soit des orifices glandulaires (fig. 3, 4, 5, 6: b1, b2 et b3).

b) Groupe apical (fig. 6):

Le groupe apical comprend huit sensilles dessinant une constellation régulière sur le côté de la cellule apicale. Quatre d'entre elles se situent en arrière du bord dorsal de la cellule apicale. Deux sont placées au niveau même de la cellule apicale ; les deux dernières s'observent latéralement aux deux précédentes (fig. 6).

\section{c) Cycles Buccaux (fig. 3, 4 et 5):}

Les cycles buccaux sont visibles par transparence dans l'entonnoir buccal, nous appelons $\mathrm{Bu} 1$ le plus externe et $\mathrm{Bu} 2$ le plus interne; ils comprennent:

Cycle Bu1: 1 Bu1D, 0 Bu1L, 0 Bu1V;

Cycle Bu2: 1 Bu2D, 0 Bu2L, 0 Bu2V.

d) Cycles labiaux (fig. 3, 4 et 5):

Les deux cycles labiaux s'observent sur la lèvre ventrale de l'entonnoir buccal, on distingue un cycle La1 interne et un cycle La2 externe. Ils comprennent:

Cycle La1: 2 La1V, 1 La1L;

Cycle La2: $1 \mathrm{La} 2 \mathrm{~V}, 0 \mathrm{La} 2 \mathrm{~L}$.

Ces cycles se prolongent en fait parmi les sensilles du groupe jugal, mais à ce niveau, il n'est plus possible de les distinguer vu la densité des sensilles jugales. C'est pourquoi nous limitons les cycles labiaux aux sensilles ventrales et latérales.

2) Zone troncale (fig. 1 et 2).

Les sensilles se répartissent comme suit dans les neuf cycles troncaux.

Cycle $\operatorname{Tr} 1$ : 1 Tr1D, $2 \operatorname{Tr} 1 \mathrm{~L}, 1 \mathrm{Tr} 1 \mathrm{~V}$,

Cycle $\operatorname{Tr} 2: 3 \operatorname{Tr} 2 \mathrm{D}, 0 \operatorname{Tr} 2 \mathrm{~L}, 0 \operatorname{Tr} 2 \mathrm{~V}$,

Cycle $\operatorname{Tr} 3: 2 \operatorname{Tr} 3 \mathrm{D}, 0 \operatorname{Tr} 3 \mathrm{~L}, 0 \operatorname{Tr} 3 \mathrm{~V}$,

Cycle Tr4: $2 \operatorname{Tr} 4 \mathrm{D}, 0 \mathrm{Tr} 4 \mathrm{~L}, 0 \mathrm{Tr} 4 \mathrm{~V}$,

Cycle $\operatorname{Tr} 5: 2 \operatorname{Tr} 5 \mathrm{D}, 2 \operatorname{Tr} 5 \mathrm{~L}, 0 \operatorname{Tr} 5 \mathrm{~V}$,
Cycle Tr6 : 0 Tr6D, 1 Tr6L, 1 Tr6V , Cycle $\operatorname{Tr} 7: 0 \operatorname{Tr} 7 \mathrm{D}, 2 \operatorname{Tr} 7 \mathrm{~L}, 1 \operatorname{Tr} 7 \mathrm{~V}$, Cycle Tr8: 1 Tr8D, 0 Tr8L, 0 Tr8V . Cycle $\operatorname{Tr} 9: 1 \operatorname{Tr} 9 \mathrm{D}, 0 \mathrm{Tr} 9 \mathrm{~L}, 0 \mathrm{Tr} 9 \mathrm{~V}$.

FIG. 7,8 et 9 . - Chétotaxie du hapteur des Polystomes européens, dessinés à la chambre claire, à la même échelle. (Nomenclature, voir légende de la fig. 1).

7. - Polystoma integerrimum. 8. - P. pelobatis. 9, - P. gallieni,

$\mathrm{Cp}$ : crochet postérieur; $\mathrm{cpl}$ : crocheton postéro-latéral; cl: crocheton latéral (1 à 6 ). 

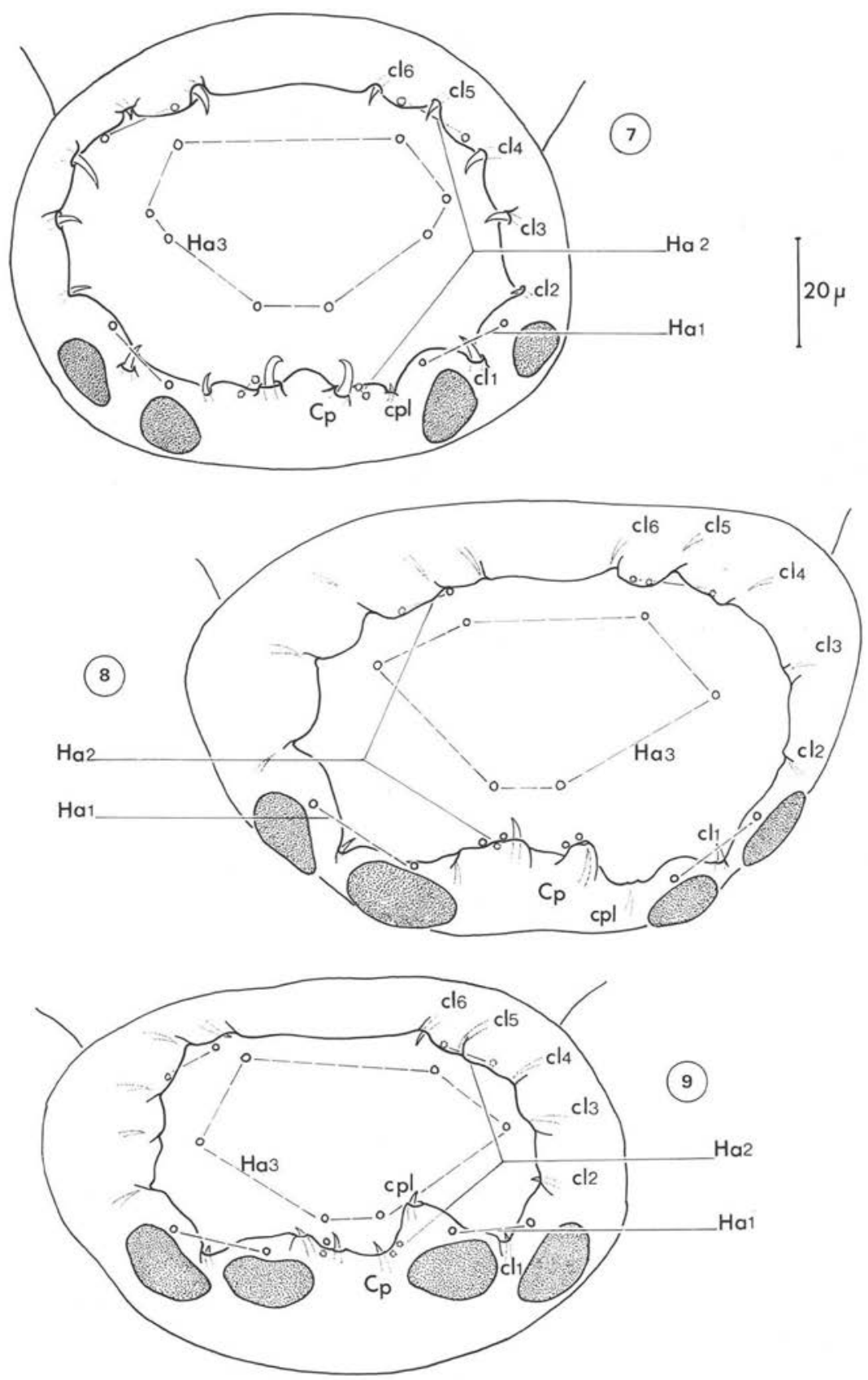
3) Zone haptoriale (fig. 7, 8 et 9).

Dans la zone haptoriale, il est possible, non seulement de numéroter les sensilles mais de préciser leur situation par rapport à l'armature (celle-ci comprend, d'arrière en avant, une paire de crochets postérieurs, une paire de crochetons postéro-latéraux et six paires de crochetons latéraux numérotés de 1 à 6) ; par contre, nous abandonnons ici les lettres indiquant la situation dorsale, latérale ou ventrale, la disposition des trones nerveux longitudinaux ne pouvant être précisée dans le hapteur.

Dans les cycles de la zone haptoriale, les sensilles sont distribuées comme suit:

Cycle Ha1: $2 \mathrm{Ha} 1$ : une entre les crochetons postéro-latéraux et latéraux 1; une entre les crochetons latéraux 1 et 2 .

Cycle $\mathrm{Ha} 2$ : $4 \mathrm{Ha} 2$ : deux entre les crochetons postérieurs et postéro-latéraux; une entre les crochetons latéraux 4 et 5 ; une entre les crochetons latéraux 5 et 6 .

Cycle $\mathrm{Ha} 3$ : $3 \mathrm{Ha} 3$ : une entre les crochets postérieurs et les crochetons postérolatéraux; une entre les crochetons latéraux 3 et 4 ; une entre les crochetons latéraux 5 et 6.

\section{Variation intraspécifique :}

Pour définir la variation intraspécifique, nous avons comparé d'une part les larves des générations 1 et 2 , d'autre part les larves d'une même génération entre elles.

\section{1) Comparaison des générations.}

Nous entendons par génération 1, l'ensemble des larves issues des œufs pondus par le Polystome adulte dans la vessie des grenouilles; nous entendons par génération 2 l'ensemble des larves issues des œufs pondus par les Polystomes dits néoténiques sur les branchies des tétards. Rappelons que ces larves ont la même morphologie et des potentialités biologiques apparemment identiques (Gallien, 1935 ; Combes, 1968).

Nous avons comparé entre elles plusieurs dizaines de larves appartenant à chacune des générations pour les espèces $P$. pelobatis et $P$. gallieni; d'un point de vue chétotaxique, nous n'avons pu relever aucune différence. Ce résultat paraît important puisqu'il confirme de façon très nette l'équivalence des deux générations larvaires.

\section{2) Comparaison des individus d'une génération.}

Comme dans le cas des cercaires de Digènes, il existe une possibilité de variation individuelle dans la disposition des sensilles des larves de Monogènes. 
La variation la plus couramment observée consiste dans l'absence d'une sensille, plus rarement dans son dédoublement. Par ailleurs, il va de soi que l'implantation d'une sensille sur le tégument oscille autour d'une position moyenne ; il en résulte que les cycles décrits sont, suivant les individus, ou bien parfaitement dessinés ou bien légèrement déformés. Les variations les plus sensibles affectent le groupe jugal. En aucun cas, cette variation individuelle n'empêche cependant l'identification des sensilles dans le cadre du système que nous avons proposé.

Il faut enfin préciser qu'on peut observer, chez certains individus, des sensilles qui n'entrent pas dans le cadre cohérent que nous avons décrit. Nous proposons de les nommer «sensilles folles». Il est possible que certaines d'entre elles aient pour origine un dédoublement suivi de dérive, mais d'autres paraissent être vraiment des éléments supplémentaires. Nous en verrons un exemple au paragraphe suivant à propos de $P$. pelobatis.

\section{Variation interspécifique :}

Les trois espèces qui nous ont servi de modèle, bien qu'étroitement spécifiques de leurs hôtes, sont morphologiquement voisines à l'état adulte: $P$. gallieni se singularise par l'absence d'anastomoses digestives préhaptoriales, tandis que $P$. integerrimum et $P$. pelobatis sont seulement distingués par le degré de ramification du tube digestif et la forme des grands crochets (hamuli) du hapteur. Par ailleurs, la disposition des cellules ciliées sur les larves (Combes, 1968) est pratiquement identique. On pouvait donc s'attendre à ce que la chétotaxie révèle à son tour une parenté très étroite.

Dans le but de mettre en évidence des variations, même discrètes, nous avons comparé attentivement 50 larves de chacune des trois espèces. Cette comparaison fait ressortir les points suivants :

a) Les cycles buccaux ne sont au nombre de deux que chez $P$. integerrimum. Chez $P$. pelobatis et $P$. gallieni un seul subsiste sans qu'il soit aisé de dire avec certitude lequel est manquant. La comparaison précise de la position des sensilles buccales des trois espèces autorise à penser cependant que le cycle qui subsiste serait Bu1 (fig. 3, 4 et 5).

b) Chez la larve de $P$. pelobatis, il existe fréquemment un petit groupe impair de sensilles folles en arrière de la cellule apicale (fig. 10). Ce groupe comporte de 3 à 5 sensilles irrégulièrement alignées; en dehors de $P$. pelobatis, nous l'avons observé une seule fois chez $P$. integerrimum, jamais chez $P$. gallieni.

c) Le hapteur de $P$. integerrimum présente beaucoup plus souvent que les deux autres espèces des sensilles folles qui viennent presque toujours s'intercaler dans le cycle $\mathrm{Ha} 3$ (fig. 7).

d) Le reste de la chétotaxie paraît identique chez les trois espèces, notamment dans la région toujours facile à analyser des taches oculaires (fig. 11) où se trouvent $2 \times 3$ sensilles ovalaires et de grande taille. 


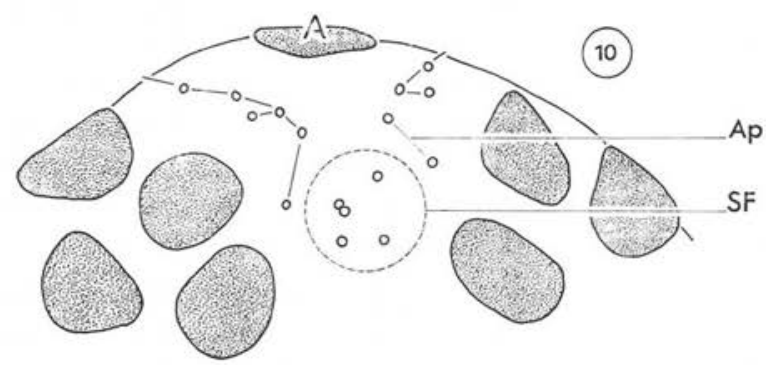

Fig. 10. - Polystoma pelobatis. Dessin à la chambre claire de la région dorso-frontale montrant une partie des sensilles du groupe apical (Ap) et des sensilles folles (SF).

- A : cellule ciliée apicale.

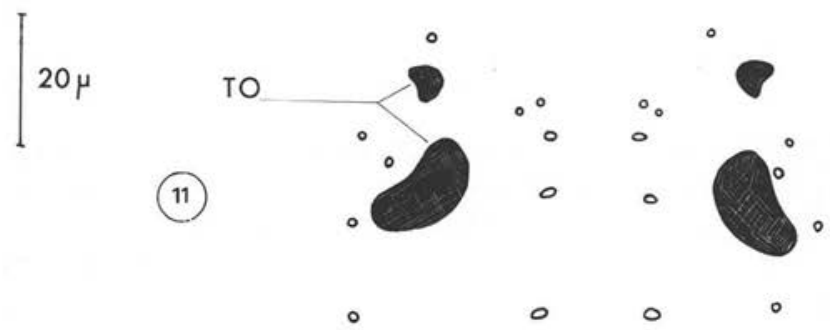

Fig. 11. - Polystoma integerrimum. Dessin à la chambre claire des sensilles dorsales au niveau des taches oculaires (T.O.).

(Les figures 10 et 11 sont à la môme échelle).

\section{Conclusion :}

Notre étude montre que, chez les Monogènes comme chez les Digènes, la chétotaxie est un élément d'identification des larves, les variations intraspécifiques étant pratiquement négligeables.

Cependant, la chétotaxie ne permet pas une identification formelle au niveau spécifique, puisque, dans la comparaison $P$. pelobatis- $P$. gallieni, nous n'avons découvert aucun caractère discriminatif constant. Il ne semble donc pas que l'on puisse, comme c'est le cas chez les cercaires, se fonder sur les sensilles des Monogènes pour séparer des espèces voisines; on ne saurait d'ailleurs oublier que la larve nageante des Monogènes n'est en aucune façon l'équivalent de la cercaire. On peut espérer que des recherches ultérieures ajouteront un intérêt phylétique à la chétotaxie des larves de Monogènes. Dans le cas précis des Polystomatidae, il sera particulièrement intéressant de comparer les larves des Polystomes africains et malgaches, à la fois entre elles et avec celles 
des Polystomes européens que nous avons décrites. Ce même intérêt s'attachera aux comparaisons entre les Polystomes néotropicaux (récemment découverts) et les Polystomes paléotropicaux.

\section{Bibliographie}

BYCHOWSKY (B. E.), 1957. - Monogenetic trematodes, their systematics and phylogeny. Akad. Nauk. U.S.S.R., 509 p. (en russe). Transl. A.I.B.S. Wash, by W. Hargis (1961).

Combes (C.), 1968. - Biologie, écologie des cycles et biogéographie de Digènes et Monogènes d'Amphibiens dans l'Est des Pyrénées. Mém. Mus. nat. Hist. nat., sér. A, 51, 1-195.

Euzet (L.) et Lambert (A.), 1971. - Compléments à l'étude de la larve de Diplozoon paradoxum Von Nordmann, 1832 (Monogenea). Ann. Parasit. hum. comp., 46, $675-684$.

- et -, 1974. - Chétotaxie comparée des larves de Diplozoon paradoxum Von Nordmann, 1832 et de Diplozoon gracile Reichenbach-Klinke, 1961. Bull. Soc. Zool. Fr., 99, 307-314.

Gallien (L.), 1935. - Recherches expérimentales sur le dimorphisme évolutif et la biologie de Polystomum integerrimum Fröl. Trav. Stat. Zool. Wimereux, 12, 1-181.

MALDER (A.-M.), 197j. - Monogènes et Trématodes parasites d'Amphibiens en Côte d'Ivoire. Rev. Suisse Zool., 80, 267-322.

OWEN (I. L.), 1970. - The oncomiracidium of the Monogenean Discocotyle sagittata. Parasitology, 61, 279-292.

Richard (J.), 1971. - La chétotaxie des cercaires. Valeur systématique et phylétique Mém. Mus. nat. Hist. nat., sér. A, 67, 1-179.

RodHe (K.), 1968. - Das Nervensystem der Gattung Polystomoides Ward, 1917 (Monogenea). Z. Morph. Tiere, 62, 58-76. 